\title{
Factores de riesgo de enfermedades cardiovasculares y conocimiento sobre complicaciones en adultos jóvenes
}

Danter Saboya Más, ${ }^{1, a}$

Saboya MD. Factores de riesgo de enfermedades cardiovasculares y conocimiento sobre complicaciones en adultos jóvenes. Cuid salud, ene-jun 2017; 3(1):257-266.

\section{RESUMEN}

Objetivo: determinar los factores de riesgo de enfermedades cardiovasculares y conocimiento sobre sus complicaciones en adultos jóvenes del Centro de Salud Micaela Bastidas de Ate-Lima. Metodología: estudio cuantitativo, diseño descriptivo, realizado en el Centro de Salud "Micaela Bastidas" de Ate, utilizando un cuestionario con las variables: factores de riesgo de enfermedades cardiovasculares-ECV y conocimiento sobre complicaciones. Participaron 235 adultos de 20-45 años de edad previo consentimiento informado. Los datos fueron analizados mediante la estadística descriptiva. Resultados: los factores de riesgo cardiovascular estaban presentes en nivel medio en los adultos jóvenes. En el análisis por dimensiones predominó también el nivel medio, el $80 \%$ tenía el factor hábitos nocivos, $71 \%$ el factor nutricional, $54 \%$ el factor actividad y $45 \%$ el factor biológico. Referente al conocimiento, el $60 \%$ tenía un nivel medio y $28 \%$ nivel alto; por dimensiones, obtuvieron nivel medio de conocimiento en complicaciones cardiológicas y no cardiológicas, aunque en conceptos generales alcanzaron nivel alto. Conclusión: existen factores de riesgo para ECV en la mayoría de los adultos jóvenes, predominando un conocimiento medio respecto a las complicaciones de ECV. Es imperativo fortalecer y operativizar eficazmente las estrategias de prevención en el primer nivel de atención conforme a las normas de la OMS.

Palabras clave: prevención de enfermedades, obesidad, tobaco, estilo de vida sedentario, enfermería de atención primaria (Fuente: DeCS BIREME).
Saboya MD. Risk factors for cardiovascular disease and knowledge of complications young adults. Cuid salud, enejun 2017; 3(1):257-266.

\begin{abstract}
Objective: To determine the risk factors of cardiovascular diseases and knowledge about their complications in young adults at the Micaela Bastidas Health Center in Ate, Lima. Methodology: Quantitative study, descriptive design, performed at the "Micaela Bastidas" Health Center of Ate-Lima, using a questionnaire that variables: risk factors of cardiovascular diseases and knowledge about complications. 235 people aged 20-45 years with informed consent participated. The data were analyzed by descriptive statistics. Results: Cardiovascular risk factors were present in the middle level in young adults. In the analysis by dimensions also prevailed the average level, $80 \%$ had harmful habits factor, $71 \%$ nutritional factor, $54 \%$ the activity factor and $45 \%$ the biological factor. Regarding knowledge, $60 \%$ were classified as medium level and $28 \%$ high; and noncardiological complications, contrary to general concepts that reached a high level. Conclusion: There are risk factors for CVD in the majority of the young adult participants, with predominance of average knowledge regarding the complications of cardiovascular diseases. It is imperative to strengthen and effectively implement prevention strategies at the first level of care in accordance with WHO standards
\end{abstract}

Keywords: Disease prevention, obesity, tobacco, sedentary lifestyle, primary care nursing (Source: MeSH NLM).

\footnotetext{
1 Hospital Nacional Dos de Mayo, Lima-Perú

a Licenciado Enfermero.
} 


\section{INTRODUCCIÓN}

La Organización Mundial de la Salud (OMS) declara que las enfermedades cardiovasculares (ECV) son la principal causa de muerte en el mundo. Cada año mueren más personas por ECV que por otras causas; siendo que el 2012 murieron 17.5 millones de personas menores de 70 años por causa de enfermedades no trasmisibles (ENT), lo que equivale al $31 \%$ de óbitos registrados en el mundo. De éstos, 7.4 millones fueron por cardiopatía coronaria y 6.7 millones por accidentes cerebrovasculares. ${ }^{1}$

Más de tres cuartas partes de esas defunciones ocurren en los países de ingresos bajos y medios. Estando proyectado que para el 2030, casi 23.6 millones de personas morirán por alguna $\mathrm{ECV}$, principalmente por cardiopatías y accidentes cerebrovasculares (ACV). ${ }^{2}$

En el Perú, la Resolución Ministerial № 771-2004MINSA, informa que en los noventa los daños no transmisibles (DNT) y, entre ellos los ACV, ocupaban las 10 primeras causas de muertes, situación que se modificó en el 2000 pasando a ocupar los cinco primero lugares. Y frente a eso, la Estrategia Sanitaria Nacional de Prevención y Control de los DNT en julio de 2004 se propone como objetivo estratégico contribuir en la disminución de la morbimortalidad por DNT., ${ }^{3,4}$

El estudio TORNASOL I que evaluó la prevalencia de factores de riesgo cardiovascular (PFRCV) como la hipertensión arterial (HTA), hipercolesterolemia, diabetes, tabaquismo, obesidad y sedentarismo; identificó que existe mayor PFRCV en la población de varones, aunque la hipercolesterolemia era mayor en mujeres, siendo más alto la prevalencia de estos factores en la Costa. ${ }^{5}$ Seis años más tarde (20102011), el estudio TORNASOL II mostró incremento significativo de la PFRCV, con excepción del tabaquismo. ${ }^{6}$

Entre enero-diciembre 2006, el primer registro nacional de infarto de miocardio agudo (RENIMA) reportó 995 casos, ocurriendo el 72,3\% en población de varones y el $47 \%$ de pacientes había presentado dos o tres factores de riesgo cardiovascular (FRCV), evidenciando que la HTA y la dislipidemia fueron los más frecuentes, cuyo cuadro se presenta cada vez en hombres más jóvenes; ${ }^{7}$ perfil que aún se mantiene con el paso de los años. ${ }^{8}$

El 2012 la mortalidad por ECV alcanzó al 22\% de la población peruana, destacando la enfermedad cerebrovascular, enfermedad isquémica del corazón, enfermedad hipertensiva y reumática, insuficiencia cardiaca, entre otras; con diferencia entre varones y mujeres. ${ }^{9}$ Situación que se torna crítica porque afecta cada vez más a la población menor de 70 años, ${ }^{9,10}$ con grave impacto en el desarrollo social y económico del país. ${ }^{11,12}$

Estudios corroboran la presencia de FRCV en diversas poblaciones, ${ }^{13-15}$ siendo preocupante su aparición en poblaciones cada vez más jóvenes. ${ }^{16-18}$ Hecho que se asocia con el sedentarismo, la diabetes, los hábitos no saludables de alimentación e hipercolesterolemia, ${ }^{19-21}$ Lo que podría estar relacionado con el grado de conocimiento que poseen las personas sobre la ECV.

Algunos estudios han identificado que las personas tienen un alto nivel de conocimiento sobre las ECV y sus complicaciones, ${ }^{22,23}$ aunque se reporta también que no conocen cuáles son los factores de riesgo mayores para desencadenar una ECV. ${ }^{24}$ En el Perú no fue identificado estudios al respecto, pero se pudo constatar que las personas tienen información poco clara sobre las ECV y sus factores de riesgo, sobre todo en la población adulta que asiste a los servicios de salud para atenderse por cualquier afección, tal es el caso del Centro de Salud Micaela Bastidas de Ate-Lima, donde existe el Programa de Control y Prevención de los DNT en cumplimiento con la Estrategia Sanitaria Nacional de Prevención y Control de ECV y diabetes en el adulto y adulto mayor. ${ }^{3}$

En tal sentido y conociendo la alta prevalencia de los FRCV en la población actual, especialmente en los países pobres y en vías de desarrollo como el Perú, además de la importancia de estar informado para asî saber tomar las debidas precauciones en el cuidado de la salud, este estudio objetivó determinar los FRCV y el conocimiento sobre sus complicaciones en adultos jóvenes que acuden al Centro de Salud Micaela Bastidas de Ate-Lima, destacando el rol de enfermería en el ámbito de la atención primaria de salud al detectar precozmente condiciones y estados prodrómicos que pueden interferir en el mantenimiento de los patrones normales de salud.

\section{MATERIAL Y MÉTODOS}

Estudio de enfoque cuantitativo y diseño descriptivo; realizado en el Centro de Salud Micaela Bastidas de Ate, de la Dirección de Salud IV Lima Este (DISA IV); cuya misión es promover estilos de vida saludables, disminuir los riesgos inherentes a enfermedades, ofrecer atención promocional y preventiva con recursos humanos calificados y comprometidos con la Institución. Brindando atención por consultorio externo en medicina, odontología, psicología, obstetricia, nutrición, farmacia, laboratorio; 
además de Control de Crecimiento y Desarrollo del Niño Sano.

La población universo fue conformada por todos los adultos jóvenes (20-45 años de edad) de ambos sexos, que asistían a consulta externa en el área de estudio; siendo estimado que acudían al Centro de Salud 1400 personas adultas jóvenes por mes, según información de la Oficina de Estadística.

Para determinar el tamaño de la muestra fue utilizado la fórmula de población finita para estudios descriptivos, considerando una confiabilidad de $95 \%$ y un margen de error de $5 \% \quad(n=235)$. La muestra seleccionada fue no probabilístico, mediante muestreo por conveniencia y según criterios de inclusión: persona de ambos sexos, con edades entre 20 y 45 años, sin diagnóstico de enfermedad cardiovascular y participación voluntaria; siendo excluidos los deportistas calificados, instructores de educación física y deporte y, aquellas con problemas de visión, audición, además de los visitante en la comunidad.

En la recolección de datos se usó la técnica de encuesta aplicando un cuestionario que constaba de tres partes: presentación, datos generales e información específica (variables en estudio), conteniendo 34 ítems en general.

Respeto a las variables, éstas fueron de tipo cualitativa y medidas por categorías. En la variable FRCV se consideró las dimensiones de riesgo: biológico, hábitos alimentarios, hábitos nocivos y desarrollo de actividad física. En conocimiento, las dimensiones fueron: conceptos generales sobre la enfermedad (ECV, causas, manifestaciones), complicaciones cardiológicas y complicaciones no cardiológicas.

En el cuestionario, 25 ítms estaban relacionadas con FRCV medidos mediante una escala de intensidad: nunca (1), algunas veces (2), casi siempre (3), siempre (4). La variable conocimiento contenía ocho ítems tomando como referente el estudio de Gamboa. ${ }^{25} \mathrm{El}$ cuestionario fue sometido a juicio de expertos con ocho peritos en el área, cuyas apreciaciones se evaluaron con el test Binomial con alta concordancia entre los jueces. Para la confiabilidad, se realizó la prueba piloto con 35 personas, quienes no formaron parte del estudio; los resultados de la variable fueron analizados mediante el test Alfa de Chronbach $(\mathrm{p}=0,7)$ y el conocimiento con Kruder y Richardson $(\mathrm{p}=0,65)$.

Para la recolección de datos, previamente se cumplió con los trámites administrativos. Pasado esta etapa, el investigador y otros dos colaboradores (previamente capacitados) hacían contacto con los sujetos de investigación en los diferentes consultorios, tópico y triaje. Este proceso fue realizado entre agosto y setiembre del 2015 en días alternos durante la semana y en horarios de 7.00-10.00 horas y de 13.00-16.00 horas.

Para aplicar el cuestionario, los sujetos de investigación debían manifestar su aquiescencia firmando la Hoja de Consentimiento Informado, además de haber sido aclarados de cualquier duda respecto al estudio, llenando las respuestas del cuestionario en un lapso de 20 minutos.

Finalizada esta etapa, los sujetos eran conducidos al área de triaje para el control de su peso y talla. Para controlar el peso se utilizó una balanza Bascula Seca de Romana, modelo 700, con capacidad de $220 \mathrm{~kg}$, el cual se ubicaba en una superficie plana colocando ambas pesas en cero y calibrándolo por cada sujeto, quien debía subir a la balanza descalzo retirando además cualquier objeto que pudiera alterar ostensiblemente su peso; ya en la balanza miraba al frente levantando la barbilla y manteniendo sus brazos extendidos a los lados del cuerpo.

La talla era medida utilizando un tallímetro de madera, el cual fue colocado a un extremo del ambiente junto a la pared invitando a subir al sujeto descalzo, solicitándole que mantenga la cabeza recta mirando al frente en ángulo de $90^{\circ}$ en función a una línea horizontal desde la punta de la nariz hasta el hueso occipital; los brazos debían mantenerse extendidos hacia abajo adheridos al cuerpo, siendo que la cabeza, espalda y los talones estaban adheridos a la madera (tallímetro).

Para el control de los datos se verificó que el cuestionario esté completamente lleno antes de recogerlo. En el análisis de datos, éstos fueron digitados primero en el programa Excel 2015, utilizando códigos para preservar el anonimato de los sujetos de investigación, etapa en que también se realizó el control de calidad de la digitación. Luego, los datos fueron transportados al programa Statistical Package for the Social Sciences (SPSS) versión 21 y procesado el análisis con la estadística descriptiva (univariables).

El proyecto fue sometido al Comité de Ética en Investigación de la Dirección de Salud IV Lima, siendo aprobado mediante carta $\mathrm{N}^{\mathrm{o}}$ 10050-2015. En la recolección de datos se tomó en cuenta los principios de ética aplicados en la investigación con seres humanos: Beneficencia, no maleficencia, Justicia y autonomía; además, los datos obtenidos fueron manejados con 
estricta confidencialidad, utilizando solo para los propósitos que se persigue en el estudio.

\section{RESULTADOS}

Casi la mitad (46\%) de los adultos jóvenes tenía entre 20-25 años de edad, seguido por los de 26-29 años (27\%); siendo el $89 \%$ (208) de sexo femenino. Respecto al peso, el $44 \%$ (104) presentaba peso normal, $44 \%$ (104) sobrepeso y $12 \%$ (28) obesidad. El gráfico 1 presenta datos referentes a la clasificación de los factores de riesgo cardiovascular.

Gráfico 1: Clasificación de los FRCV en adultos de 2045 años de edad. Centro de Salud Micaela Bastidas de Ate, setiembre 2015.

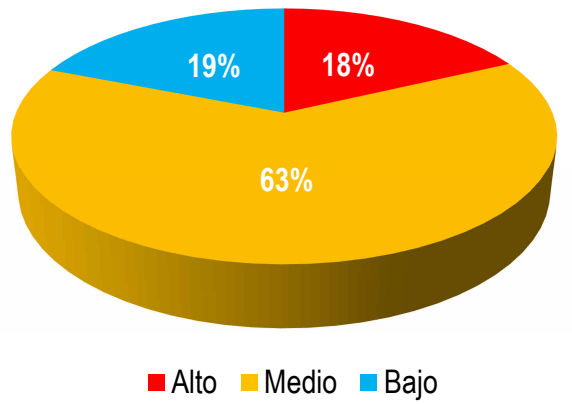

En el gráfico 1, el 63\% (149) de los adultos jóvenes encuestados fue clasificado dentro de riesgo cardiovascular de nivel medio y el $18 \%$ (41) en alto, este último con más de tres factores presentes al momento de la entrevista: hábitos nocivos, nutricional, biológicos y sedentarismo.

Gráfico 2: FRCV por dimensiones en adultos de 20-45 años de edad. Centro de Salud Micaela Bastidas de Ate, setiembre 2015.

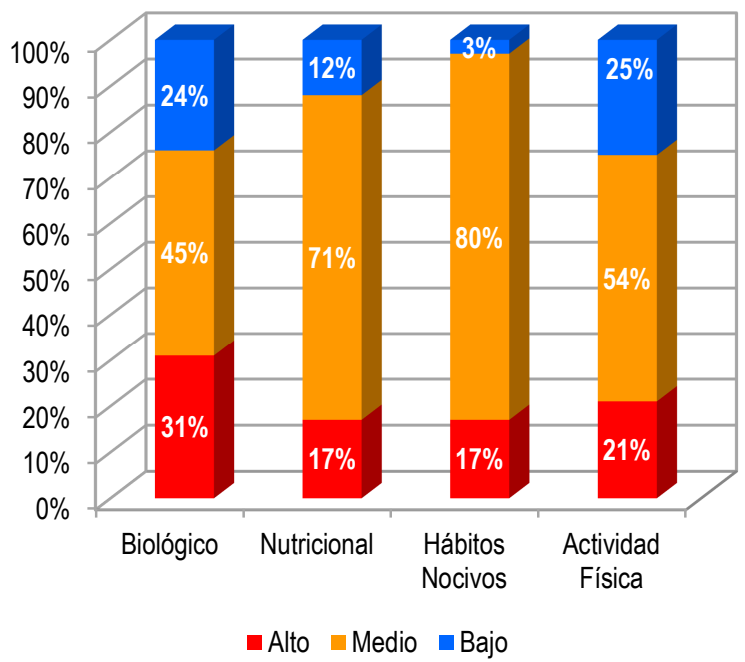

En el gráfico 2, los factores riesgo nutricional y hábitos nocivos presentaron mayores porcentajes en el nivel medio $(71 \%=166$ y $80 \%=188$ respectivamente), mientras que en el factor biológico un importante porcentaje de adultos jóvenes presentó alto riesgo considerando los antecedentes familiares y el IMC; que, sumado con el nivel medio este factor llega al $76 \%$. En actividad física, casi el $80 \%$ se encontraba entre media y baja frecuencia de realizar algún deporte, ejercicios o asistir al gimnasio.

Propiamente, en el gráfico 3, más de un tercio $(39 \%=90)$ de adultos jóvenes presentó antecedentes familiares para enfermedad cardiovascular y la mayoría $(56 \%=131)$ se clasificó en riesgo mediante su IMC (sobrepeso y obesidad).

Gráfico 3: FRCV biológicos en adultos de 20-45 años de edad. Centro de Salud Micaela Bastidas de Ate, setiembre 2015 .

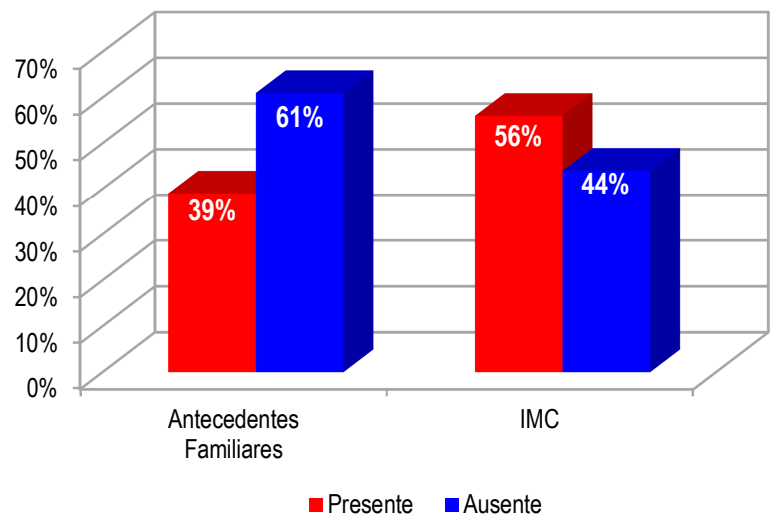

Gráfico 4: FRCV nutricionales en adultos de 20-45 años de edad. Centro de Salud Micaela Bastidas de Ate, setiembre 2015.

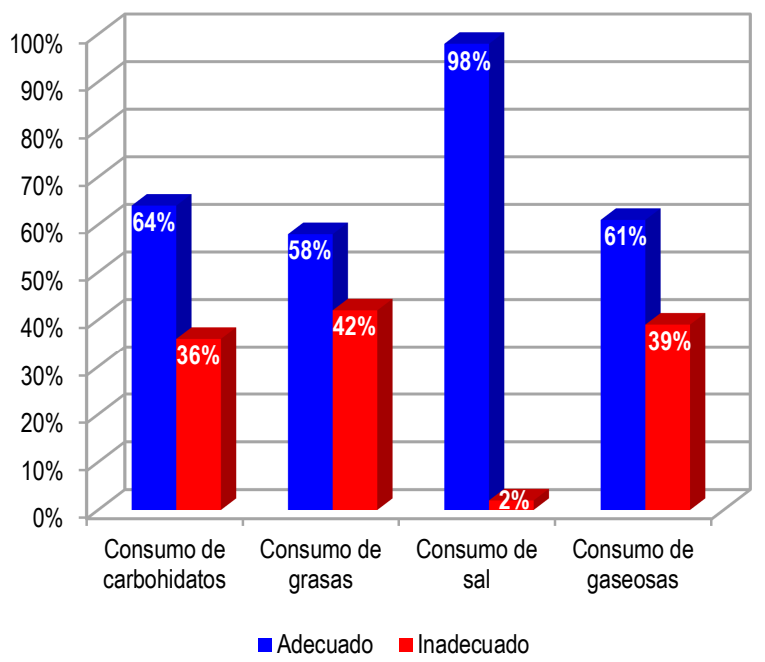


Respecto a los hábitos nutricionales, más de un tercio de adultos jóvenes presentó hábitos inadecuados en su alimentación, especialmente en consumo de alimentos con alto contenido de grasa $(42 \%=98)$, bebidas gaseosas $(39 \%=92)$ y carbohidratos $(39 \%=85)$, excepto por el exceso de sal en los alimentos (gráfico 4).

Gráfico 5: FRCV nocivos en adultos de 20-45 años de edad. Centro de Salud Micaela Bastidas de Ate, setiembre 2015 .

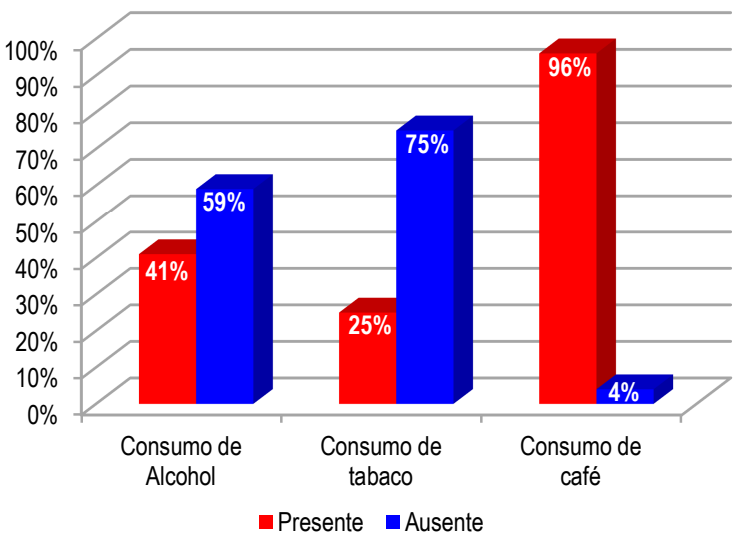

En los hábitos nocivos, se observa un alto porcentaje de consumo de café $(96 \%=225)$, seguido de bebidas alcohólicas $(41 \%=96)$ y de tabaco $(25 \%=58)$, aunque estos datos no reflejan necesariamente una alta frecuencia durante la semana, ya que fue medido utilizando una escala de intensidad (gráfico 5). Asimismo, en el gráfico 6, sobre el desarrollo de actividad física de forma cotidiana, entendiendo ésta como la realización de cualquier actividad deportiva o semejante; predomina la práctica inadecuada $(57 \%=134)$, con una duración menor de 60 minutos por vez $(57 \%=134)$ y al menos una vez a la semana o ninguna $(56 \%=132)$.

Gráfico 6: Distribución de los FRCV actividad física en adultos de 20-45 años de edad. Centro de Salud Micaela Bastidas de Ate, setiembre, 2015.

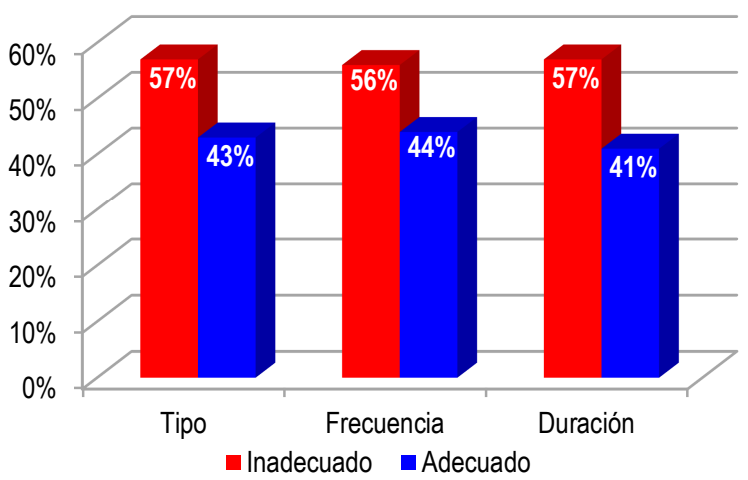

Gráfico 7: Conocimiento sobre complicaciones en ECV en adultos de 20-45 años de edad. Centro de Salud Micaela Bastidas de Ate, setiembre 2015.

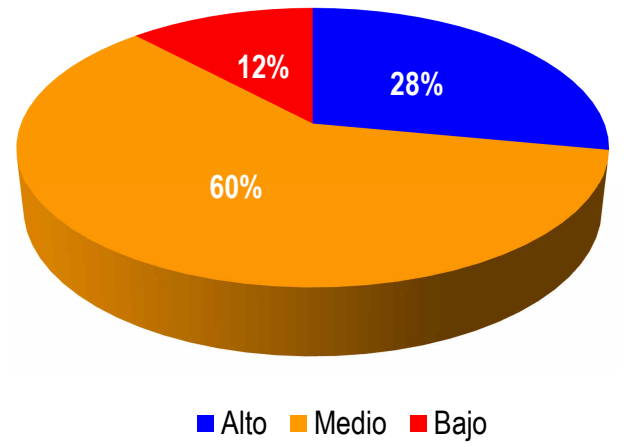

Gráfico 8: Conocimiento sobre complicaciones de ECV por dimensiones, en adultos de 20-45 años de edad. Centro de Salud Micaela Bastidas de Ate, setiembre 2015.

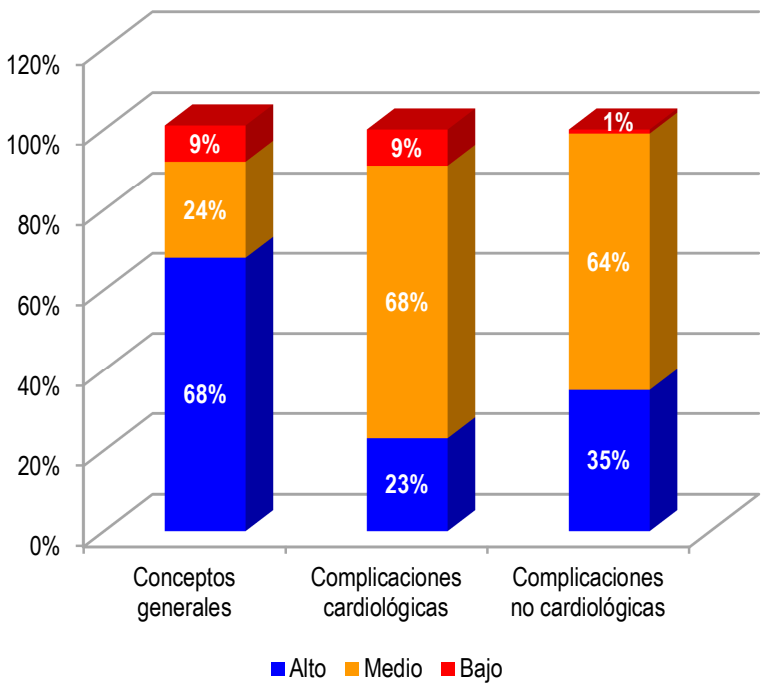

En el gráfico 7 se destaca en los adultos jóvenes el nivel medio $(60 \%=141)$ y bajo $(12 \%=28)$ de conocimiento sobre complicaciones de ECV. Siendo que en el análisis por dimensiones (gráfico 8) predominó también el conocimiento medio en complicaciones cardiológicas $(68 \%=161)$ y no cardiológica $(64 \%=150)$.

\section{DISCUSIÓN}

Está demostrado que el cese de hábitos nocivos, la reducción de sal en la dieta, el consumo de frutas y hortalizas y la actividad física regular reducen el riesgo de ECV; en tal sentido, es imperativo crear entornos propicios y establecer políticas sanitarias que aseguren la asequibilidad y disponibilidad de servicios y espacios que fomenten una vida saludable. ${ }^{1}$ 
En el estudio, más del $80 \%$ de adultos era menor de 35 años de edad y casi la mitad presentaba sobrepeso y $12 \%$ obesidad. Aguilar y Cácere ${ }^{20}$ identificaron en su estudio que el $39,8 \%$ era obeso. ${ }^{20}$ Situación que indica al sobrepeso y la obesidad como FRCV en importantes, debido al consumo excesivo de comidas con escaso valor nutritivo, alta concentración de grasa saturadas y de carbohidratos, lo que también se puede corroborar en este estudio (gráfico 4).

Los malos hábitos de vida agregados al factor biológico constituyen FRCV que finalmente desencadenan algún tipo de enfermedad cardiovascular, 19-21 hecho preocupante cuando se trata de personas jóvenes ${ }^{16-18}$ por las consecuencias discapacitantes que trae a la salud a lo largo de la vida. En el estudio el factor biológico o antecedente familiar y el índice de masa corporal elevado estaban presentes en alta proporción (gráfico 3).

Actualmente, en el Perú el sobrepeso y la obesidad constituyen un problema de salud pública en la población, con mayor prevalencia en Lima, ${ }^{5-8,14}$ ciudad que alberga a casi 10 millones de habitantes. ${ }^{26} \mathrm{La}$ OMS recomienda la urgencia de cambio en los estilos de vida de la población, dado que es mucho más económico establecer pautas y estrategias multisectoriales para controlar y reducir el impacto socioeconómico que provocarán las enfermedades no trasmisibles hacia el $2025 .{ }^{27}$

Los datos del gráfico 1 muestran una alta proporción de adultos jóvenes con FRCV presentes (sumando nivel medio y alto), destacando el antecedente biológico y la escasa actividad física que realizan, pues la clasificación alto y medio significan que los participantes tenían una vida más sedentaria que activa (gráfico 2), lo que puede ser observado más al detalle en el gráfico 6 respecto a tipo, frecuencia y duración de los ejercicios o deportes. Estos resultados son conducentes con el estudio de Bezerra et al. ${ }^{18}$ realizado en una población escolar de 20-24 años de edad al norte de Ceará- Brasil.

Según la OMS, ${ }^{27}$ el $23 \%$ de adultos $\geq 18$ años de edad no era lo suficientemente activo en el 2014, siendo las mujeres menos activas que los hombres y las personas mayores lo eran menos que los jóvenes. Destacando que el riesgo de muerte por cualquier causa es más elevado en los adultos cuya actividad física es insuficiente, que entre aquellos que practican al menos 150 minutos de ejercicio físico moderado por semana; reduciendo el riesgo de cardiopatía isquémica, accidente cerebrovascular, diabetes, cáncer de mama y de colon.
Sobre los hábitos nocivos, la gran mayoría en el estudio se clasificó entre riesgo medio y alto (gráfico 2), reportando consumo de café, alcohol y tabaco en orden secuencial de mayor consumo (gráfico 5). Un estudio de revisión concluye que el consumo de café hasta 300 $\mathrm{ml} /$ día o menos representa un factor de protección y puede ser hasta beneficioso para la salud; pasado esta cantidad su asociación con las ECV está demostrado aunque los estudios difieren en la precisión de cantidades. ${ }^{28}$ Situación que aún amerita ser investigado y en la que debe considerarse las características biológicas de cada persona. ${ }^{29}$

El consumo de alcohol y tabaco como FRCV vienen siendo evaluados en varios estudios con población adulta joven. ${ }^{16-18}$ Respecto al alcohol, su consumo supera el $70 \%$ en la población entre hombres y mujeres, ${ }^{16,18}$ casi duplicando el porcentaje identificado en este estudio (gráfico 5). Sobre el tabaco, los estudios reportan un consumo de $10-17 \%,{ }^{16,17}$ porcentajes menores al identificado en el estudio (25\%) posiblemente porque la edad de los participantes llega a 40 años. En Colombia, estudio realizado el 2012 indica que los hábitos nocivos se inician antes de los 20 años de edad y se hace más efectivo entre los 23 y 24 años. ${ }^{30}$ En el Perú, el 20\% de la población mayor de 15 años consume tabaco y $90,2 \%$ consume alcohol, destacando a Lima Metropolitana como el área dónde se consume más, secundado por la Selva. ${ }^{20,31,32}$

La OMS establece que los países deben aplicar políticas severas para la venta y publicidad del alcohol y tabaco en las sociedades, con una reducción progresiva de su consumo en la población a lo largo de los años, debido a su relación directa con la muerte de millones de personas anualmente en el mundo por causa de las enfermedades no transmisibles, incluyendo las ECV. Políticas que son enarboladas por los sistemas de salud a través de normas y programas; sin embargo, existe poca o ninguna monitorización, educación y sensibilización a la población en los establecimientos de salud. Gomes et al. ${ }^{18}$ refieren que disponer de información de manera eficiente para vivir saludablemente sería altamente económico, el 83,5\% de su población desconocía o no participaba en actividades educativas para prevenir enfermedades cardiovasculares en los últimos 12 meses.

En el estudio, el conocimiento sobre ECV y sus complicaciones fue de nivel medio a alto (gráfico 7), con mejor información sobre la enfermedad, sus causas, signos y síntomas; respecto a las complicaciones cardiológicas y no cardiológicas hubo un predominio medio (gráfico 8). Lo que demuestra que estas personas 
podrían estar más alertas y atentas a cualquier indicio precoz de los problemas cardiovasculares como lo refieren Gomes et al. ${ }^{18}$

Contrariamente, los adultos jóvenes del estudio parecen no prestar atención a esas alertas, posiblemente porque no estan conscientes del daño y de las consecuencias que pueden sufrir por estas enfermedades; algunos de sus comentarios reflejan que tienen el concepto erróneo de que estar gordito es tener buena salud; están más pendientes en atender las tareas del hogar, trabajar y resolver los problemas diarios con la familia que orientar sus acciones al cuidado de la salud. En consecuencia, solo asisten al Centro de Salud cuando sienten un fuerte malestar o cuando se encuentran totalmente enfermos.

Datos que corroboran los resultados de estudios previos que indagaron sobre conocimiento de los FRCV y su evolución crónica en personas no enfermas. ${ }^{22-24} \mathrm{Si}$ bien en el área de estudio existe el programa de la Estrategia Sanitaria Nacional de Prevención y Control de Enfermedades no Transmisibles, la misma no está del todo implementada y los profesionales tampoco están sensibilizados para cumplir la meta establecida por la OMS; no existe seguimiento ni monitoreo de los pacientes diagnosticados y mucho menos de los no diagnosticados.

La información que poseen los adultos jóvenes en el estudio fueron adquiridos de forma ocasional a través de los medios de comunicación masiva, como la televisión, radio y redes sociales, que como se ve, no llega a concientizar a la persona para tomar una actitud y comportamiento de cambio. Estudios reflejan que la educación en los servicios de salud debe ser constante y reiterativa para llegar a producir cambios de comportamiento. $^{19,20,33}$

En este sentido, el Ministerio de Salud debe asumir con responsabilidad el compromiso con la OMS sobre la prevención y reducción de las ENT en el país, el primer paso son las políticas y normas de los cuáles ya se dispone, pero aún falta organizar, capacitar y sensibilizar al personal de salud y ofrecer la infraestructura suficiente para implementar y ejecutar esta gran tarea que significa cuidar la salud de la población para la prevención. En necesario aumentar el recurso humano en el primer nivel de atención, sobre todo el personal de enfermería, quien tiene suficientes competencias y habilidades para ejecutar acciones de promoción y prevención en la comunidad y en todos los niveles de atención. Es urgente invertir y redireccionar recursos financieros desde los órganos centrales para operativizar los programas de prevención, especialmente porque vale más prevenir y concientizar a la población sobre la importancia de llevar una vida saludable, que curar y tratar en los hospitales o enfrentar una invalidez temprana de las personas, con el elevado costo que esto implica para la familia y el país.

La teoría de Nola Pender sobre el modelo Promoción de la Salud, se orienta hacia factores cognitivos-perceptuales de las personas partiendo de la vivencia cotidiana y de sus relaciones con la familia y con el entorno, donde el profesional de enfermería se constituye en un ente facilitador que reorienta los modos de vida, preferencias, concientizando la necesidad de cambio para desarrollar modos de vida saludables, contando con la participación de la familia y de grupos que se fortalezcan entre sí mismos por los logros alcanzados. $^{34}$

Este modelo facilita la identificación de conceptos relevantes sobre conductas de promoción de salud, basándose en la educación de las personas sobre cómo cuidarse y cómo llevar una vida saludable. La teoría de Nola Pender continúa siendo perfeccionada y ampliada en cuanto a su capacidad para explicar las relaciones entre los factores que se cree influye en las modificaciones de la conducta sanitaria. ${ }^{34}$

Entre las limitaciones del estudio, éstas se relacionan básicamente a su muestreo no probabilística que imposibilita la generalización de los resultados, además que el centro de salud fue elegido sin considerar la prevalencia de los FRCV en ese distrito limeño. Siendo necesario profundizar el tema en otras poblaciones considerando la relevancia de la prevención y sensibilización de la población frente a la grave amenaza que constituyen las enfermedades cardiovasculares, dado que cada vez más afecta a la población más joven y que la sociedad actual asume cada vez más fácil un estilo de vida de riesgo, por las largas horas de trabajo, las dietas insanas, poca a nula actividad física, escazas áreas de esparcimiento y deporte, entre otros.

En conclusión, el estudio muestra presencia de diversos factores de riesgo para desarrollar enfermedades cardiovasculares como: hábitos nocivos, nutricional, actividad física y biológico. En el factor hábitos nocivos se identificó consumo de alcohol, tabaco y café a temprana edad; en el nutricional, existe un consumo excesivo de carbohidratos, grasas y comida rápida con alto contenido de frituras, harina, sal y colorantes; en la actividad física, solo un porcentaje mínimo realiza algún tipo de ejercicio físico adicional a sus actividades de rutina. Los adultos jóvenes tienen conocimiento de medio a alto sobre complicaciones de 
las ECV, lo que pudo evidenciarse en las dimensiones conceptos generales, complicaciones cardiológicas y no cardiológicas. Realidad que demanda actividades de fortalecimiento y funcionamiento adecuado del programa de Estrategia Sanitaria Nacional de Prevención y Control de Enfermedades no Transmisibles; además, se de concientizar y sensibilizar a las personas que, a pesar de tener conocimiento sobre la enfermedad, hacen caso omiso al potencial desarrollo de la enfermedad, complicaciones y sus consecuencias.

\section{Correspondencia:}

Danter Saboya Más

Correo electrónico: danter_saboya@ hotmail.com

\section{REFERENCIAS BIBLIOGRÁFICAS}

1. Organización Mundial de la Salud-OMS. Enfermedades Cardiovasculares: Centro de prensa [Internet]. Ginebra: OMS; ene 2015 [actualizado ene 2015; citado 5 mar 2016]. Disponible en: http://www.who.int/mediacentre/factsheets/fs317/es/

2. Organización Mundial de la Salud-OMS. Enfermedades Cardiovasculares:¿Qué son las enfermedades cardiovasculars? [Internet]. Ginebra: OMS; s.f. [actualizado s.f.; citado 5 mar 2016]. Disponible en: http://www.who.int/cardiovascular diseases/about c $\mathrm{vd} / \mathrm{es} /$

3. Ministerio de Salud. Resolución Ministerial $N^{\circ}$ 7212005/MINSA. Dirección General de Salud de las Personas. Plan General Estrategia Sanitaria Nacional de Prevención y Control de los Daños no Transmisibles. 2004-2012 [Internet]. Lima-Perú; Minsiterio de Salud; 2012 [citado 06 abr 2016]. p.26. Disponible en: ftp://ftp2.minsa.gob.pe/normaslegales/2005/RM7212005.pdf

4. Ministerio de Salud. Información de Mortalidad [Internet]. Lima-Perú: Ministerio de Salud; s.f. [citado 24 jul 2016]. p.22. Disponible en: http://www.minsa.gob.pe/estadisticas/estadisticas/Sa laSituacional/04_Mortalidad.pdf

5. Segura VL, Agusti CR, Parodi RJ. Factores de Riesgo de las Enfermedades Cardiovasculares en el Perú (Estudio Tornasol). Rev Peruana de Cardiología [serie en internet]. May-agos 2006 [citado 24 jul de 2016]; 32(2):82-83. Disponible en: http://sisbib.unmsm.edu.pe/BVRevistas/cardiologia/ v32_n2/pdf/a02.pdf
Declaración de financiamiento y de conflictos de interés:

El estudio fue financiado por el autor, declara no tener conflictos de interés.

\section{Contribución de auditoría:}

Saboya MD: Concepción y diseño del estudio, recolección de los datos, análisis e interpretación de los resultados y aprobación de la versión que será publicada

6. Segura VL, Agusti CR, Ruiz ME. Factores de Riesgo de las Enfermedades Cardiovasculares en el Perú II. Rev Peruana de Cardiología [serie de internet]. Ene-abr 2013 [citado 24 jul 2016]; 39(1)5$57 . \quad$ Disponible en: http://repebis.upch.edu.pe/articulos/rpc/v39n1/a1.pdf

7. Reyes RM, Heredia LJ, Campodónico HS, Drago SJ, Alvarado CO. Registro Nacional de Infarto de Miocardio Agudo (REMIMA). Rev. Peruana de Cardiología. [serie de internet]. May-ago 2008 [citado 24 jul 2016]; 34 (2): 85-98. Disponible en: http://sisbib.unmsm.edu.pe/bvrevistas/cardiologia/v3 4_n2/pdf/a02v34n2.pdf

8. Reyes RM, Ruiz ME. Registro Nacional de Infarto de Miocardio Agudo II REMIMA II. Rev Peruana de Cardiología [serie de internet]. Ene-abr 2013 [citado 24 jul 2016]; 39 (1): 60-70. Disponible en: http://repebis.upch.edu.pe/articulos/rpc/v39n1/a2.pdf

9. Organización Mundial de la Salud. Enfermedades no transmisibles: perfiles de países 2014 (Perú). [internet]. Ginebra: OMS; 2014 [citado 10 jul 2015]. p.02.

Disponible: http://www.who.int/nmh/countries/per_es.pdf

10. Jorstad HT, Colkesen EB, Boekholdt SM, Tijssen JG, Wareham NJ, Peters R, et al. Estimated 10-year cardiovascular mortality seriously underestimates overall cardiovascular risk. Heart [serie de internet]. Sep 2015 [citado 24 jul 2016]; (0):1-6. Disponible en:

http://heart.bmj.com/content/heartjnl/early/2015/08/ 10/heartjnl-2015-307668.full.pdf

11. Organización Panamericana de la Salud/Organización Mundial de la Salud. 28 Conferencia Sanitaria panamericana. 64 ${ }^{\mathrm{a}}$ Comité Regional. Estrategias para la prevención control de 
las enfermedades no trasmisibles [internet]. OPS/OMS: Washington CD: sep 2012 [citado 24 jul 2016].

Disponible: http://www.paho.org/hq/index.php?option=com_doc $\underline{\text { man\&task}=\text { doc_view\&gid }=19267 \& \text { Itemid }}$

12. McCarthy K, Long-biao C, Fang-rong X, Pei-gang W, Hong-liang X, Yong-ling Y, et al. Urban-rural differences in cardiovascular disease risk factors: a cross-sectional study of schoolchildren in Wuhan, China. PloS ONE [serie de interent]. Sep 2015 [citado 10 jul 2016]; 10 (9):1-12. Disponible en: https://www.ncbi.nlm.nih.gov/pmc/articles/PMC456 $\underline{4102 /}$

13. Alonso DJA, Calleja MAB, Borbolla RS. Prevalencia de los factores de riesgo cardiovascular en trabajadores de una planta metalúrgica en Santander España. Rev. Med Segur Trab [serie de internet]. Jul-set 2012 [citado 14 oct 2014]; 58(228): 269-281.Disponible.

en: http://gesdoc.isciii.es/gesdoccontroller?action=down load\&id=28/11/2012-f654ea5ae7

14. Ruiz ME, Segura VL, Agusti CR. Uso del Score de Framingham como Indicador de los Factores de Riesgo de las Enfermedades Cardiovasculares en la Población Peruana. Rev Peruana de Cardiología [serie de internet]. Set-dic 2012 [citado 4 oct 2014]; 38(3):1-19. Disponible en: http://www.revespcardiol.org/contenidos/static/prem io_cardio/revista-peruana-cardiologia.pdf

15. García IA, Lozano AJE, Álamo SR, Vega AT. Evolución de los factores de riesgo cardiovascular entre 2004 y 2009 en la cohorte del estudio del riesgo de enfermedad cardiovascular en Castilla y León (RECCyL). Angiología [serie de internet]. Julago 2015 [citado 01 ago 2016]; 67(4):259-265. Disponible en: http://www.elsevier.es/es-revistaangiologia-294-articulo-evolucion-los-factores-

riesgo-cardiovascular$\underline{\text { S0003317014002934? redirectNew }=\text { true }}$

16. García-Gulfo MH, García-Zea JA. Prevalencia de factores de riesgo cardiovascular en Jóvenes de una institución universitaria. Rev salud pública [serie de internet]. Mar-jul 2012 [citado 4 oct 2014]; 14(5):822-830. Disponible en: http://www.scielosp.org/pdf/rsap/v14n5/v14n5a09.p df

17. Zemdegs JCS, Corsi LB, Coelho LDC, Pimentel GD, Hirai AT, Sachs A. Lipid profile and cardiovascular risk factors among first-year Brazilian university students in Sao Paulo. Rev Nutrición Hospitalaria [serie de internet]. May-jun 2011 [citado 14 oct 2014]; 26(3):553-559.
Disponible en: http://scielo.isciii.es/pdf/nh/v26n3/18_original_14.p df

18. Gomes EB, Moreira MTM, Pereira HCV, Sales IB, Lima FIT, Freitas CHA et al. Factores de riesgo cardiovascular en adultos jóvenes de una ciudad del nordeste de Brasil. Rev bras enferm [serie de internet]. Jul-ago 2012 [citado 6 oct. 2014]; 65(4):594-600. Disponible en: http://www.scielo.br/scielo.php?script=sci_arttext\&p

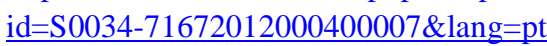

19. Sifuentes CA, Sosa GE, Pérez MAR, Parra FFM. Riesgo cardiovascular del personal de enfermería en el área quirúrgica. Enferm Glob [serie de internet]. 2011 [citado 14 oct 2014]; 10 (21):1-10. Disponible en:

http://revistas.um.es/eglobal/article/view/115181/10 $\underline{9111}$

20. Aguilar CY, Cáceres GP. Prevalencia y factores de riesgo asociados a hipertensión arterial, Hospital José Agurto Tello, Chosica. Rev Facultad de Medicina Humana. [serie de internet]. 2013 [citado 5 mar 2016]; 13(1):26-32. Disponible en: http://aulavirtual1.urp.edu.pe/ojs/index.php/RFMH/a rticle/view/164

21. Adrianzen BChA, Villegas ASLM. Descripción de factores de riesgo cardiovascular modificable de pacientes adultos del consultorio de nutrición del Hospital Nacional Guillermo Almenara Irigoyen [tesis de licenciatura]. [internet]. Lima: Universidad Peruana de Ciencias Aplicadas; 2014 [citado 11 nov 2014]. Disponible en: http://repositorioacademico.upc.edu.pe/upc/bitstrea m/10757/322282/2/adrianzen bc-pub-tesis.pdf

22. Pérez PRD, Rodríguez LM. Nivel de conocimientos sobre hipertensión arterial, de pacientes atendidos en un Centro de Diagnóstico Integral de Venezuela. ccm [serie de internet]. Jul-set 2015 [citado 03 Set 2016]; 19(3):406-417 disponible en: http://scielo.sld.cu/pdf/ccm/v19n3/ccm03315.pdf

23. Díaz CS, Gonzales MF, Arrieta VK, Alvis TJ, Bertel MMI. Conocimiento y presencia de factores de riesgo cardiovasculares en estudiantes de odontología de la Universidad de Cartagena [tesis de pregrado]. [internet]. Cartagena: Universidad de Cartagena de Colombia; 2013 [citado 03 de set 2016]. p.72. Disponible en: http://190.242.62.234:8080/jspui/bitstream/11227/26 $\underline{03 / 1 /-}$ \%20CONOCIMIENTOS $\% 20$ Y\%20PRESENCIA $\%$ 20DE\%20FACTORES\%20DE\%20RIESGO\%20CA 
RDIOVASCULAR\%20EN\%20ESTUDIANTES\%2 0DE\%20ODONTOLOGIA\%20FI.pdf

24. Guerrero DIG. Conocimiento de los Factores de Riesgos Mayores en la Enfermedad Cardiovascular de la Población de Estudiantes, Profesores y Administrativos de la Facultad de Ciencias Económicas y Administrativas de la Pontificia Universidad Javeriana, Bogotá D.C. [tesis de pregrado]. [internet]. Bogota: Pontificia Universidad Javeriana de Bogota; 2014 [citado 03 de set 2016]. Disponible en: http://repository.javeriana.edu.co/bitstream/10554/1 5489/1/GuerreroDiazIvanGerardo2014.pdf

25. Gamboa V. Factores de riesgo y conocimiento de la hipertensión arterial en adolescentes de la Institución Educativa María Reiche de Newman [tesis de pregrado]. Lima: Universidad Ricardo Palma; 2013. p.78.

26. Instituto Nacional de Estadística e Informática (INEI): Perú Síntesis estadística 2015 [internet]. Lima: INEI; set 2015 [citado 20 de jul 2016]. p.105. Disponible:

https://www.inei.gob.pe/media/MenuRecursivo/publ icaciones_digitales/Est/Lib1292/libro.pdf

27. Organización Mundial de la Salud: Informe sobre la situación mundial de las enfermedades no trasmisibles 2014 (resumen de orientación) [internet]. OMS: 2014 [citado 20 de jul 2016].p.16. Disponible:

http://apps.who.int/iris/bitstream/10665/149296/1/W HO_NMH_NVI_15.1_spa.pdf

28. Valenzuela BA. El café y sus efectos en la salud cardiovascular y en la salud materna. Rev Chil Nutr [serie de internet]. Dic 2010 [citado 20 jul 2015]; 37(4): 514-23. Disponible: http://www.scielo.cl/pdf/rchnut/v37n4/art13.pdf

29. Figueroa VWM. Café y enfermedades cardiovasculares. Atención primaria [serie de internet]. Nov 2009 [citado 20 jul 2015]; 41(11): 633-6. Disponible en: http://www.sciencedirect.com/science/article/pii/S02 $\underline{12656709004545}$ [Base de datos Elservier]

30. Comunidad Andina. Proyecto PRADICAN. Unión Europea. II Estudio Epidemiológico Andino sobre consumo de drogas en la población universitaria. Informe Colombia, 2012 [internet]. Lima-Peru: Secretaría General de la Comunidad Andina; ene 2013 [citado 6 mar 2016]. p.98. Disponible en: http://www.comunidadandina.org/StaticFiles/20134 2915590informe_colombia2012.pdf

31. Instituto Nacional de Estadística e Informática. Perú Enfermedades no transmisibles y transmisibles, 2014 [internet]. Lima-Perú: INEI; abr 2015 [citado 8 mar 2016]. p.144. Disponible en: https://www.inei.gob.pe/media/MenuRecursivo/publ icaciones_digitales/Est/Lib1212/Libro.pdf

32. Presidencia del Consejo de Ministros. Comisión nacional para el desarrollo y Vida Sin Drogas DEVIDA. Estrategia Nacional de Lucha contra las drogas 2012-2016: [internet]. Lima: PCM, DEVIDA; feb 2012 [citado 8 mar 2016]. p.65. Disponible en: http://www2.congreso.gob.pe/sicr/cendocbib/con4_u ibd.nsf/8FE34E42B623D47805257CAF00528280/\$ FILE/Estrategia_Nacional_Lucha_ContraDrogas_20 12_2016.pdf

33. Norris LS, Lau J, Smith JS, Schmid HC, Engelgau MM. Self-Management Education for Adults With Type 2 Diabetes. A meta-analysis of the effect on glycemic control. [serie de internet]. Jul 2002 [citado 17 oct 2015]; 25(7): 1159-1171. Disponible en: http://care.diabetesjournals.org/content/diacare/25/7/ 1159.full.pdf

34. Raile AM, Marriner TA. Modelos y teorías en enfermería. 7a ed. Madrid: Elsevier. España; 2011. 